\title{
Photogrammetric methods of terrain and obstacle data collection and AMDB creation based on satellite and UAS imagery
}

\author{
Liudmila Mitsevich ${ }^{1, *}$, and Natalia Zhukovskaya ${ }^{2}$ \\ ${ }^{1}$ Moscow State University of Geodesy and Carthography, Faculty of Applied Cosmonautics and \\ Photogrammetry, Photogrammetry Department, 4 Gorokhovsky per., 105064 Moscow, Russian \\ Federation \\ ${ }^{2}$ Belarusian State University, Faculty of Geography, Soil Science and Land Information Systems \\ Department, 16 Leningradskaya st., 220030 Minsk, Republic of Belarus
}

\begin{abstract}
The paper discusses the photogrammetric methods and process of creating geodata for aeronautical information databases. Efficient technology for collecting data on terrain, obstacles and cartographic information using satellite and aerial photos is given. The measurement, selection and evaluation of spatial data have met the international requirements for the completeness and accuracy as well as the developed structure of the attribute data base. The advantages of digital photogrammetric system and geographic information systems as the main software for aeronautical data gathering, are considered.
\end{abstract}

\section{Introduction}

The development of aeronautical information database has become particularly relevant due to high-intensity aircraft movement and requirements increasing for the air traffic safety. Aeronautical data is data used for aeronautical applications such as navigation, flight planning, flight simulators, terrain awareness, and other purposes. Terrain and obstacles data as well as aerodrome mapping database (AMDB), are essential parts of aeronautical data. There are international normative documents developed by ICAO (International Civil Aviation Organization), EUROCONTROL and other [1-7], which regulate the basic and special requirements for the collection and management of aerodrome geospatial data.

The traditional data collection technology is a terrestrial survey using geodetic GNSS (Global Navigation Satellite Systems) equipment. When studying the aeronautical information specifics, the requirements for providing data, the published data accuracy, the geographical location of airports, it becomes obvious that geodetic methods for terrain and obstacles data collecting are not always effective [3]. They require considerable efforts to restore the geometry features from coordinate sets. The normative documents provide an assessment of the geodetic method of obstacles measuring as one possible, but extremely laborious. Aerodromes are often located in intensive development areas or covered with thick forest, where terrestrial methods for determining objects heights and shapes are very difficult.

\footnotetext{
*Corresponding author: mluda789@gmail.com
} 
The article discusses the AMDB creation by photogrammetric methods in the PHOTOMOD digital photogrammetric system using ultra-high resolution satellite images and aerial photography from unmanned aircraft systems (UAS) equipped with GNSS. Data determined by several sources are mutually checked, supplemented and controlled. Replacing ground-based surveys with photogrammetric works allows to create a reliable 3D model of hard-to-reach areas (forests, mountain areas), to carry out the process of collecting, processing and measuring data virtually and to combine all data in one geo-information space.

In photogrammetric processing, automatic and semi-automatic methods for creating and filtering 3D models are used, calculations of obstacle exceedances above the digital elevation model (DEM) detection surface are performed. Then the three-dimensional topologically related vectorization of features is carried out. The advantages of using aerial survey materials are feature and attribute data completeness, unlimited visual and software evaluation of obstacles and mapping features, stereo measurements and mutual control of geospatial data.

\section{Preparatory work}

The process of geospatial data collection for near-aerodrome and aerodrome areas includes preparatory work, terrain data creating, obstacles data measuring, and the aerodrome elements mapping. In the near-aerodrome area, zones are identified. For each zone (area) different requirements on the data accuracy and detailing are imposed (Fig. 1).
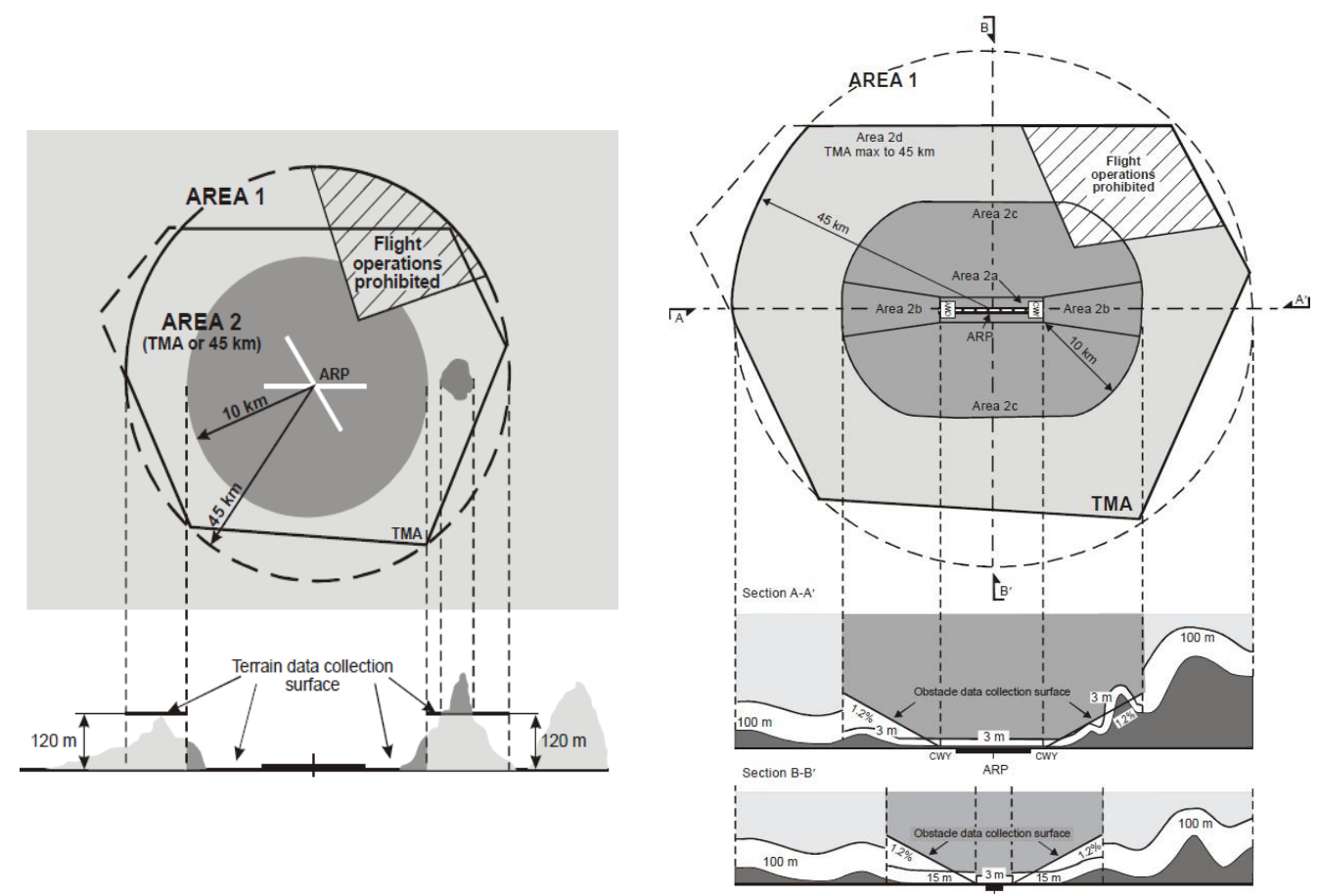

Fig. 1. Terrain and obstacle data collection areas. Source: [2]

Preparatory work includes documentations and standards study, exploration of the geographical features, ordering and acquisition of satellite and aerial imagery, aerial triangulation. Based on the materials studied, a number of objects are created in 
PHOTOMOD. These are vector and raster 2D and 3D models i.e. obstacle detection surfaces, identified obstacles, cartographic layers, AMDB with attribute information.

The exploration of the geographical features (topography, vegetation, climate features) within areas 2-4 of the aerodrome territory (Fig. 1) determines the composition and scope of work, as well as the selection of the required remote sensing data. For example, the required horizontal accuracy for the area 2 is $5 \mathrm{~m}$, the vertical accuracy is $3 \mathrm{~m}$. In the absence within $2 \mathrm{~d}$ area territories exceeding $120 \mathrm{~m}$ above the height of the aerodrome reference point (ARP), the requirements for the area 2 are reduced to the requirements for the area 1 which are $50 \mathrm{~m}$ and $30 \mathrm{~m}$ respectively. In this case, a digital terrain model can be created using remote sensing data of 5-10m resolution or ready-made terrain models (for example, SRTM). If there are digital topographic maps of 1:100 000 scale and larger for the territory it is advisable to use them to get appropriate digital elevation model (DEM). For all options for DEM creating, control based on available reliable sources and datasets is required.

To ensure the required accuracy for area 2 , it is necessary to use a stereo pair of satellite images with a resolution of near $0.5 \mathrm{~m}$ (WorldView 3, 4; Pleiades A, B). When planning it should be considered seasonality and individual weather indicators of the airdrome territory. Requirements for images are the absence of clouds, precipitation and stable snow cover. For satellite imagery geolocation, it is advisable to perform preliminarily aerial surveys using wings-type UAS in compliance with the photogrammetric rules (image overlap of $60 \%$ per $40 \%$ etc.).

UAS $0.05-0.15 \mathrm{~m}$ resolution data is simultaneously used to ensure the mapping information and digital terrain models (DTM) accuracy within the area 3-4 (areas 3, 4 are located at the aerodrome movement territory) [2]. To control AMDB in GIS and platforms for viewing aeronautical data, the orthophoto maps created from UAS data are used. According to the requirements for geodetic accuracy of aerodrome mapping information $(0.25-0.5 \mathrm{~m})$, UAS must be equipped with GNSS receiver, with the photographic centers coordinates being determined. It is also advisable to use UAS imagery in case of cloudiness in remote sensing data and hard-to-reach territory within area 2. The highest (critical) requirements are imposed on the accuracy of providing the Runway Thresholds coordinates: 1/100 arc seconds, which is about $0.3 \mathrm{~m}$ for the territories of European states. The aerial triangulation report is used to determine RMS and maximum residuals. So, while final $\mathrm{RMS}=0.1 \mathrm{~m}$ and $\mathrm{MAX}=0.25 \mathrm{~m}$, the guaranteed accuracy of the output map data in the stereoscopic model will be $0.25 \mathrm{~m}$. The processing of space imagery and the subsequent stereo vectorization of the airfield elements is performed in the digital photogrammetric system PHOTOMOD or similar software (INFO, ERDAS IMAGINE).

All geodetic horizontal measurements must be made at WGS-84 (World Geodetic System) or ITRS 2005 (International Terrestrial Reference System of the 2005 year) as required in DOC 9674 [6]. All vertical absolute heights should be given at EGM 96 (Earth Gravitational Model of the 1996 year) or more precise the national gravitational model. In that case, originators of data have to describe parameters of heights transformation from national model to EGM 96.

\section{Terrain and obstacle data collection}

To create terrain data (DEM / DTM), a stereo pair of satellite images or aerial UAS data is used, depending on the area. The resolution of the DEM must match the resolution of the source images. To obtain the "bare earth"/DTM, various types of vegetation and buildings filtering are used: by raster tone, by height difference, etc. If necessary, the resulting 3D surface model is used as the primary source for identifying height obstacles. 
Obstacles data is the most important piece of information used in air traffic safety management. If the obstacle is in the air movement zone, it must be removed (tree vegetation must be cut down). Therefore, the observation of obstacles is performed with the necessary periodicity for the aerodromes.

Detection and measurement of obstacles by photogrammetric methods are carried out by creating detection surfaces to identify and limit obstacles (Fig. 2).

Preliminarily, in the stereo pair of images, the surface model is vectorized according to specified parameters: distance from runway thresholds, location from the outer lines, heights and angles of inclination (Fig. 2a). According to the vector model, the raster detection surfaces are created (internal horizontal surface, surface of the interrupted landing, etc.) (Fig. 2b). The horizontal and vertical coordinates of the existing obstacles are imported into a separate vector layer. Changes are tracked in stereo mode, the composition and appearance of new obstacles are analyzed. Newly appeared obstacles: forest vegetation, capital construction objects, etc. are detected in the case of intersection with the detection surface (Fig. 2c). Obstacles that do not cross the surface have the negative value. All positive measurements are fixed in the control report (Fig. 2d).

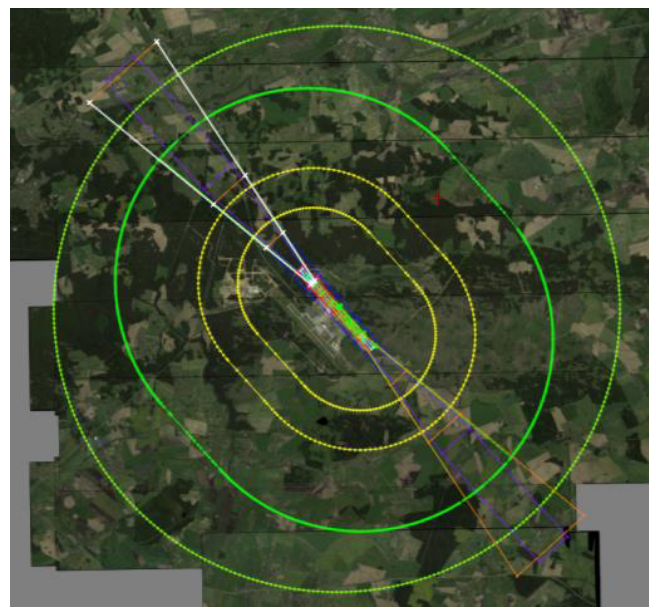

a) Vectorized detection surfaces

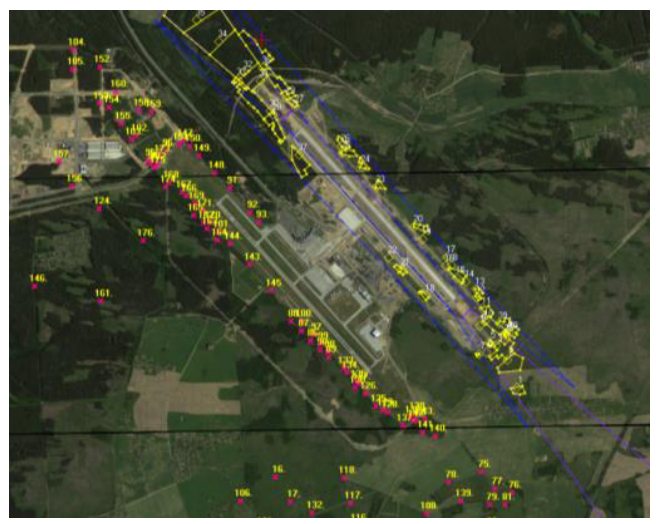

c) Obstacles stereo identification

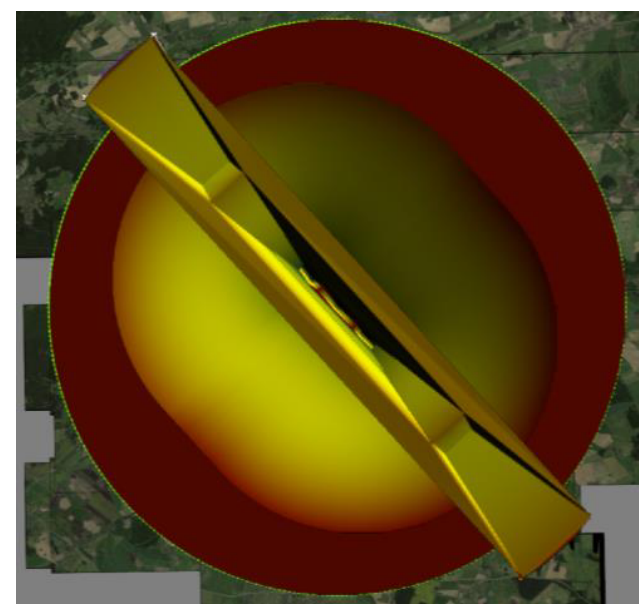

b) Raster detection surfaces

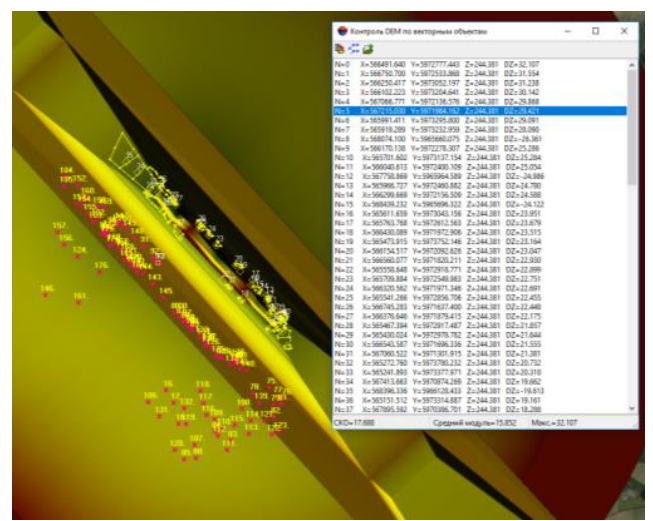

d) Obstacles hights definition above the detection surfaces

Fig. 2. Identify and evaluate obstacles on the detection surfaces. Source: authors 
The required vertical accuracy of identifying obstacles for region 2 is $3 \mathrm{~m}$, so $0.5 \mathrm{~m}$ of satellite images resolution is sufficient enough to identify and control obstacles in the area of $2 \mathrm{~b}, 2 \mathrm{c}, 2 \mathrm{~d}$. Obstacles in the area $2 \mathrm{a}$ and 3 (radio navigation communications, beacons, approach lights) are determined using UAS data. Area 4 is an obstacle free zone. High productivity obstacles stereo detecting methods, as well as the possibility of unlimited visual control, exclude data loss.

\section{AMDB data creation}

The final stage of aerodrome geospatial data collection is aerodrome mapping database creation. AMDB is a geospatial database, which describes digital geometric and semantic characteristics of aerodrome elements. The database contains detailed information about aerodrome elements (navigation elements, runways, taxiways lines, lighting equipment, buildings etc.). The requirements for the geodetic accuracy of aerodrome elements geometry are $0.5 \mathrm{~m}$. To form AMDB features, stereo pairs or orthophoto maps created using the UAS data are applied. Aerodrome feature attributes (semantics) are collected in compliance to the requirements of international aeronautical data exchange standards $[4,5]$. The feature attribute database structure (classifier) is developed in GIS PANORAMA (Fig. 3).

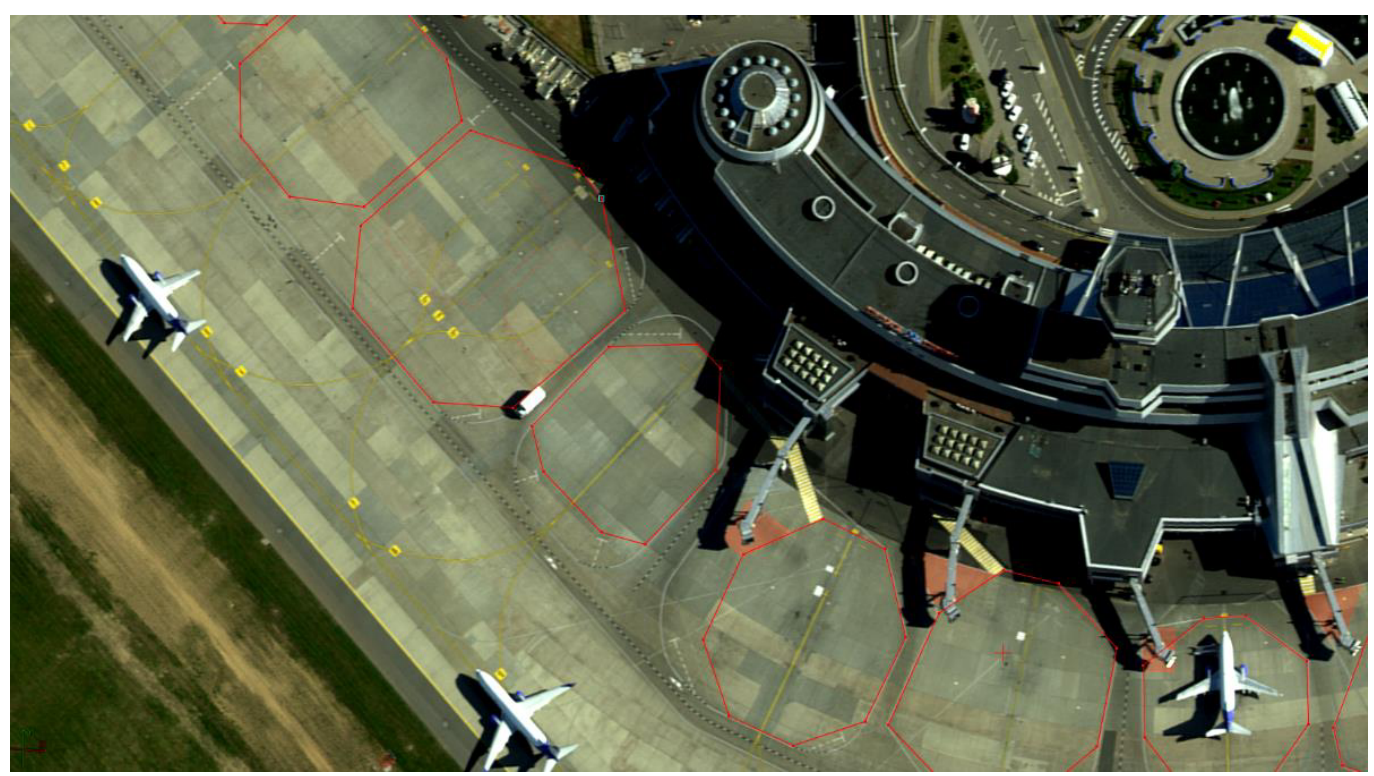

Fig. 3. Creating objects of the Parking Stand Area layer. Source: authors

The feature topology is defined by aeronautical standards and serves for the safety of aircrafts movement within the aerodrome area. The vectorization of AMDB layers in stereo provides topological connectivity of features and the possibility of their joint editing. Monitoring of the AMDB data is based on the terrestrial geodetic control.

\section{Conclusion}

The remote and cost effective technology of terrain, obstacle and AMDB data collection by photogrammetric methods, meets the requirements of European and international standards for the quality of aeronautical information. The main sources of geodata are satellite and aerial 
stereo imagery based on the GNSS equipment, which provides a centimeter accuracy. To implement this technology, the digital photogrammetric system PHOTOMOD and GIS PANORAMA are used as the main software. The advantages of photogrammetric methods are completeness, accuracy, remote geodata collection, mutual virtual monitoring of aeronautical data sets.

\section{References}

1. ICAO Annex 14: International Standards and Recommended Practices Aerodromes Volume I - Aerodrome Design and Operations. 6th edition, ICAO, 2016.

2. ICAO Annex 15: International Standards and Recommended Practices - Aeronautical Information Services. 15th edition, ICAO, 2016.

3. EUROCONTROL Terrain and Obstacle Data Manual. Edition: 2.1 Edition date: November 2015 Reference nr: EUROCONTROL-GUID-0158.

4. RTCA DO-272C/EUROCAE ED-99C: User Requirements for Aerodrome Mapping Information. Sept. 28, 2011.

5. RTCA DO-291C/EUROCAE ED-99C: Interchange Standards for Terrain, Obstacle, and Aerodrome Mapping Data. Sept. 22, 2015.

6. WORLD GEODETIC SYSTEM-1984 (WGS-84) Manual 2nd - Edition (2002) (DOC 9674).

7. RTCA D0-201A, Standards for Aeronautical Information. Prepared by SC, April 19, 2000. 